\title{
Nitric oxide production by Peromyscus yucatanicus (Rodentia) infected with Leishmania (Leishmania) mexicana
}

\author{
Elsy Nalleli Loría-Cervera $/{ }^{+}$, Erika Ivett Sosa-Bibiano, \\ Liliana Estefanía Villanueva-Lizama, Nicole Raymonde Van Wynsberghe, \\ Silvia Beatriz Canto-Lara, José Luis Batún-Cutz, Fernando José Andrade-Narváez
}

Laboratorio de Inmunología, Centro de Investigaciones Regionales Dr Hideyo Noguchi, Universidad Autónoma de Yucatán, Yucatán, México

Peromyscus yucatanicus (Rodentia: Cricetidae) is a primary reservoir of Leishmania (Leishmania) mexicana (Kinetoplastida: Trypanosomatidae). Nitric oxide (NO) generally plays a crucial role in the containment and elimination of Leishmania. The aim of this study was to determine the amount of NO produced by P. yucatanicus infected with L. (L.) mexicana. Subclinical and clinical infections were established in P. yucatanicus through inoculation with $1 \times 10^{2}$ and $2.5 \times 10^{6}$ promastigotes, respectively. Peritoneal macrophages were cultured alone or co-cultured with lymphocytes with or without soluble Leishmania antigen. The level of NO production was determined using the Griess reaction. The amount of NO produced was significantly higher $(p \leq 0.0001)$ in co-cultured macrophages and lymphocytes than in macrophages cultured alone. No differences in NO production were found between $\mathrm{P}$. yucatanicus with subclinical L. (L.) mexicana infections and animals with clinical infections. These results support the hypothesis that the immunological mechanisms of NO production in $\mathrm{P}$. yucatanicus are similar to those described in mouse models of leishmaniasis and, despite NO production, P. yucatanicus is unable to clear the parasite infection.

Key words: nitric oxide - Peromyscus yucatanicus - Leishmania (L.) mexicana

Cutaneous leishmaniasis (CL) caused by Leishmania (Leishmania) mexicana Biagi, 1953, emend. Garham, 1962, is a seasonal wild zoonosis that is endemic in the Yucatan Peninsula, Mexico (Andrade-Narváez et al. 2003). In this area, CL, known as "chiclero's ulcer", produces a single ulcer in $84.5 \%$ of patients and is commonly (39.9\%) located on the ear lobe (Seidelin 1912, Canto-Lara et al. 1998, Andrade-Narváez et al. 2001). The annual incidence rate of symptomatic infection, i.e., $\mathrm{CL}$, in the state of Campeche is $0.5 \%$, whereas the subclinical infection prevalence rate is 19\% (AndradeNarváez et al. 1990, Arjona-Villicaña 2002).

In Campeche, three rodent species have been identified as primary reservoirs of $L$. (L.) mexicana: Ototylomys phyllotis Merriam, 1901; Peromyscus yucatanicus JA Allen \& Chapman, 1897, and Heteromys gaumeri JA Allen $\&$ Chapman, 1897. The two latter species are endemic to the Yucatan Peninsula (Chable-Santos et al. 1995, CantoLara et al. 1999, Van Wynsberghe et al. 2000, 2009).

$P$. yucatanicus has been adapted to captivity and a colony has been established for experimental studies in our institution. Previous studies have demonstrated that $P$. yucatanicus experimentally infected with $1 \times 10^{6} \mathrm{~L}$. (L.) mexicana promastigotes showed clinical and histological features similar to those of humans with CL caused by the

Financial support: CONACYT (SEP-2004-COI-46390)

+ Corresponding author: nalleli.cervera@uady.mx

Received 31 May 2012

Accepted 14 September 2012 same parasite species (Sosa-Bibiano et al. 2012). These results support the utility of $P$. yucatanicus as a novel experimental model to study CL caused by $L$. (L.) mexicana.

In the laboratory mouse (Mus musculus Linnaeus, 1758) model of CL, the resistance of C57BL/6 mice to Leishmania (Leishmania) major infection has been associated with the classical activation of macrophages. Activated macrophages express inducible nitric oxide (NO) synthase and the up-regulation of this gene induces the synthesis of NO from L-arginine in a two-step process, with hydroxyl-arginine and citrulline as intermediates. NO is a potent cytotoxin involved in the killing of Leishmania parasites and therefore it is a key molecule in the control of the disease (Stenger et al. 1994, Fang 1997, Bogdan et al. 2000).

In 1976, Preston and Dumonde, based on a study about asymptomatic or subclinical infections in humans caused by $L$. (L.) mexicana, demonstrated that low doses $\left(10^{2}, 10^{3}\right)$ of Leishmania (Leishmania) tropica induced subclinical infection in M. musculus (González \& Biagi 1968, Preston \& Dumonde 1976). Subsequently, it was demonstrated that the parasite dose determines the $\mathrm{Th}_{1} /$ $\mathrm{Th}_{2}$ nature of the response to $L$. (L.) major independent of the infection route or the strain of the host or parasite (Bretscher et al. 1992, Doherty \& Coffman 1996, Menon \& Brestcher 1998).

Inbred laboratory strains of mice have been helpful in elucidating the cell types, cytokines, signal transduction cascades and antileishmanial effector mechanisms involved in the clinical resolution and progression of disease. Although these experimental models have had the major advantage of allowing control over the genetics of both the parasite and the host, they do not recapitulate the pathology observed in human disease. 
A new approach to study host-parasite relationships has been the use of wild animals, particularly primary reservoirs, as experimental animal models. Wild animals are similar to humans in that they are genetically polymorphic and thus represent an emerging system for the genetic analysis of the physiological and behavioural bases of habitat adaptation (Guénet \& Bonhomme 2003).

Laboratory studies using natural hosts as experimental models of Leishmania could increase our understanding of the mechanisms involved in immune activation during nonpathogenic and pathogenic infections. The study of dogs, the main reservoir of visceral leishmaniasis, has led to increased interest in the immune responses and the Leishmania antigens implicated in protective cellular immunity in canine visceral leishmaniasis (Alvar et al. 2004, Baneth et al. 2008).

The possible role of NO in $P$. yucatanicus infected by $L$. (L.) mexicana remains unknown. Therefore, as part of our continuing effort to develop P. yucatanicus as a novel experimental animal model to study CL caused by $L$. (L.) mexicana, in the present study, we determined the amount of NO produced by peritoneal macrophages and macrophages and lymphocytes co-cultured in the presence or absence of soluble Leishmania antigen (SLA). The macrophages and lymphocytes were obtained from $P$. yucatanicus animals that were clinically or subclinically infected with $L$. (L.) mexicana.

\section{MATERIALS AND METHODS}

Animals - Wild P. yucatanicus animals adapted well to captivity and a colony derived from wild progenitors captured in the state of Campeche has been maintained for experimental studies in our institution since 1998. Six-18-month-old P. yucatanicus animals of both sexes were selected. The animals were maintained in the animal care facility of the Regional Research Centre of the University of Yucatan. The mice were individually housed in small cages $(19 \times 29 \times 12 \mathrm{~cm})$ lined with wood shavings and were fed rodent chow ad libitum (2018S Harlan, Wisconsin). For enrichment and food balance, the mice were given fresh fruit or vegetables once per week. The animals were kept at $22^{\circ} \mathrm{C} \pm 1^{\circ} \mathrm{C}$ with a $12 / 12$ $\mathrm{h}$ light cycle. Physical enrichment was provided weekly in the form of cardboard tubes for use as hiding places and soft paper for use as nesting material. The animals were euthanised with an overdose of sodium pentobarbital $(100 \mathrm{mg} / \mathrm{kg} \mathrm{IV})$.

Parasites and antigen - The $L$. (L.) mexicana MHET/ MX/97/Hd18 strain was selected and its infectivity was restored by passage in Syrian golden hamsters (Chable-Santos et al. 1995, Canto-Lara et al. 1999, Van Wynsberghe et al. 2000, Dumonteil et al. 2003). Promastigotes were grown in Senekjie medium for seven days at $23^{\circ} \mathrm{C}$. Stationary phase promastigotes were washed three times in RPMI-1640 (RPMI medium, Gibco) before being counted and adjusted to the concentrations needed for inoculation. SLA was obtained from stationary promastigotes that were washed twice in phosphate buffered saline (PBS), resuspended in PBS and phenylmethanesulfonyl fluoride and subjected to five freeze-thaw cycles at $-70^{\circ} \mathrm{C}$ and $37^{\circ} \mathrm{C}$. The protein content was determined by the Bradford method. The final concentration of SLA used for the in vitro stimulation of the co-cultures was $2.5 \mu \mathrm{g} / \mathrm{mL}$.

Experimental infection - To study NO production, three groups of 14 P. yucatanicus mice were inoculated in the base of the tail as follows: Group 1, RPMI medium (control), Group 2, $1 \times 10^{2}$ promastigotes of $L$. (L.) mexicana (subclinical group), and Group 3, $2.5 \times 10^{6}$ promastigotes of $L$. (L.) mexicana (clinical group). The evolution of the infection was followed up weekly for 12 weeks. All animals were examined for the following clinical signs that are suggestive of Leishmania infection: oedema, erythema, ulcers and scars.

L. (L.) mexicana $k D N A$ detection in subclinically infected animals by polymerase chain reaction (PCR) - To verify the subclinical infection, another group of $22 P . y u-$ catanicus mice inoculated with $1 \times 10^{2} \mathrm{~L}$. (L.) mexicana promastigotes were sacrificed at 18 weeks post-infection and biopsy samples were obtained from the skin for parasite DNA detection by PCR. Briefly, DNA was extracted from tissue biopsy samples after homogenisation with $100 \mathrm{mM}$ Tris-HCl, $\mathrm{pH} 8.0,100 \mathrm{mM}$ ethylenediamine tetraacetic acid (EDTA), $100 \mathrm{mM} \mathrm{NaCl}, 1 \%$ sodium dodecyl sulfate and $4 \mu \mathrm{L}$ of $10 \mathrm{mg} / \mathrm{mL}$ proteinase $\mathrm{K}$ (Sigma). The DNA precipitate was dissolved in $200 \mu \mathrm{L}$ of Tris-EDTA. Total DNA (300 ng) was amplified in $100 \mathrm{mM} \mathrm{KCl}, 20$ $\mathrm{mM}$ Tris- $\mathrm{HCl}, 0.1 \mathrm{mM}$ EDTA, $1 \mathrm{mM}$ dithiothreitol and $1.75 \mathrm{mM} \mathrm{MgCl}$ in the presence of $0.2 \mathrm{mM}$ of each deoxyribonucleotide, 10 pmol of each primer and 2.5 units of Taq DNA polymerase in a final volume of $25 \mu \mathrm{L}$. Primers 13A (5'GTGGGGGAGGGGCGTTCT3') and 13B (5'ATTTTACACCAACCCCCAGTT3') were used to amplify a conserved region of the kinetoplast DNA (kDNA) minicircle (120 bp) of L.(L.) mexicana (Kerr et al. 2006). The positive PCR control was DNA extracted from a logarithmically growing culture of $L$. (L.) mexicana strain MHET/MX/97/Hd18 and the negative control was the PCR mixture without DNA. PCR products were separated by electrophoresis in 1\% agarose gels in trisborate-EDTA (45 mM tris-borate, $1 \mathrm{mM}$ EDTA).

Macrophages and lymphocytes - All P. yucatanicus mice included in the NO experiments were inoculated intraperitoneally with incomplete Freund's adjuvant 10 days before being euthanised. Macrophages were obtained by peritoneal cavity lavage with RPMI. The cells were washed three times in RPMI and their viability was determined by the trypan blue exclusion test. Macrophages were adjusted to $1 \times 10^{5} / \mathrm{mL}$ in complete culture medium containing RPMI supplemented with $10 \%$ foetal bovine serum, $1 \%$ non-essential amino acids, $0.1 \% \beta$-mercaptoethanol and $100 \mathrm{U} / \mathrm{mL}$ penicillinstreptomycin. Splenocytes were obtained by maceration, washed three times with RPMI and depleted of erythrocytes by treatment with lysis solution (Promega Z3141). Macrophages were separated from lymphocytes by adherence to glass dishes at $37^{\circ} \mathrm{C}$ at $5 \% \mathrm{CO}_{2}$ for $2 \mathrm{~h}$. Lymphocytes were washed in RPMI and adjusted to $1 \times 10^{6}$ in complete culture medium. Macrophages alone or in co-culture with autologous lymphocytes were plated in 
24-well flat-bottom culture plates with RPMI. Another co-culture was stimulated with $2.5 \mu \mathrm{g} / \mathrm{mL}$ SLA. All groups were incubated at $37^{\circ} \mathrm{C}$ at $5 \% \mathrm{CO}_{2}$ for $96 \mathrm{~h}$. The in vitro experiments were repeated once.

NO quantification - The culture supernatants were collected and the amount of NO produced was indirectly determined by the quantification of the nitrite $\left(\mathrm{NO}_{2}^{-}\right)$ concentration using Griess reagent. Briefly, after $96 \mathrm{~h}$ of incubation, cell culture supernatants from macrophages were mixed with an equal volume of Griess reagent and the absorbance was spectrophotometrically measured at $540 \mathrm{~nm}$. The $\mathrm{NO}_{2}{ }^{-}$concentration was determined using a standard $\mathrm{NaNO}_{2}$ curve and was expressed as $\mu \mathrm{M} / \mathrm{mL}$.

Statistical analysis - Analysis of variance (one-way ANOVA) with a p-value $<0.05$ was used to compare the levels of NO production among the three culture types (peritoneal macrophages alone and co-cultures of macrophages and autologous lymphocytes with or without SLA stimulation) and among the doses of infection: no parasite (control), $1 \times 10^{2}$ promastigotes of $L$. (L.) mexicana (subclinical infection) and $2.5 \times 10^{6}$ (clinical infection).

Ethics - All animals were handled according to the Mexican Law for the use of laboratory animals (fmvz. unam.mx/fmvz/principal/archivos/062ZOO.PDF) and the Guide for the Care and Use of Laboratory Animals (NRC 1996) and the proposal was approved by our institutional bioethics committee.

\section{RESULTS}

Experimental infection - Subclinical infection was induced in $100 \%$ of $P$. yucatanicus mice inoculated with $1 \times 10^{2} L$. (L.) mexicana parasites and $92.3 \%(12 / 13)$ of rodents inoculated with $2.5 \times 10^{6}$ parasites developed one or more suggestive signs of CL beginning at two weeks post-infection. The most frequent symptoms were oedema, induration, ulcers and, later, scars at the inoculation site. No lesions were found in the control group inoculated with RPMI.

TABLE

Nitric oxide productions in all groups

\begin{tabular}{|c|c|c|c|}
\hline Groups & Stimulus & Mean & SD \\
\hline \multirow[t]{3}{*}{ Healthy } & MФs & 0.5553 & 0.51833 \\
\hline & Co-culture & $0.8835^{a}$ & 0.57388 \\
\hline & Co-culture/SLA & $1.1064^{a}$ & 0.58228 \\
\hline \multirow[t]{3}{*}{ Subclinical } & MФs & 0.8159 & 0.36438 \\
\hline & Co-culture & $1.1321^{a}$ & 0.55636 \\
\hline & Co-culture/SLA & $1.4287^{a}$ & 0.39042 \\
\hline \multirow[t]{3}{*}{ Clinical } & MФs & 0.6521 & 0.43567 \\
\hline & Co-culture & $1.0058^{a}$ & 0.31240 \\
\hline & Co-culture/SLA & $1.2500^{a}$ & 0.48972 \\
\hline
\end{tabular}

$a: \mathrm{p}<0.05$. Two samples were analyzed for each animal. Fourteen animals per group were used. Results are expressed as means \pm standard deviation (SD). SLA: Leishmania antigen.
L. (L.) mexicana $k D N A$ detection in subclinically infected animals by PCR - The PCR results were positive for 12 of the 22 P. yucatanicus (54.5\%) mice inoculated with $1 \times 10^{2} L$. (L.) mexicana promastigotes.

NO production - The level of NO production was significantly higher $(\mathrm{p} \leq 0.0001)$ in co-cultured macrophages and lymphocytes than in the monocultured macrophages for all three groups of P. yucatanicus (Table). The level of NO production was significantly higher $(\mathrm{p} \leq 0.05)$ in subclinically infected $P$. yucatanicus mice than control mice (Fig. 1). No differences in NO production were found between P. yucatanicus mice inoculated with $1 \times 10^{2}$ parasites and those inoculated with $2.5 \times 10^{6}$ parasites at 12 weeks post-infection (Fig. 2).

\section{DISCUSSION}

In the present study, we determined that $\mathrm{NO}$ was produced by peritoneal macrophages and by co-cultured macrophages and lymphocytes from $P$. yucatanicus mice infected with $L$. (L.) mexicana and grown in the

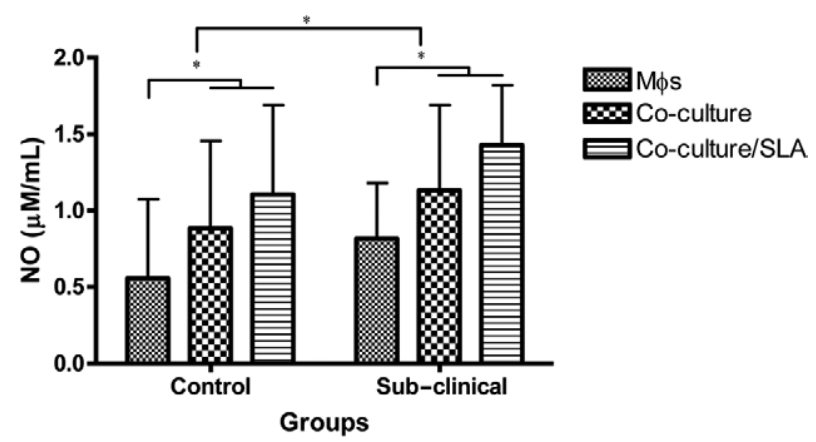

Fig. 1: nitric oxide (NO) productions by control and sub-clinical infected Peromyscus yucatanicus. Data in sub-clinical rodents were registered at 12 week post-infection. Two samples were analysed for each animal. Fourteen animals per group were used. Results are expressed as means \pm standard deviation. Asterisks mean $p<0.05$. SLA: Leishmania antigen.

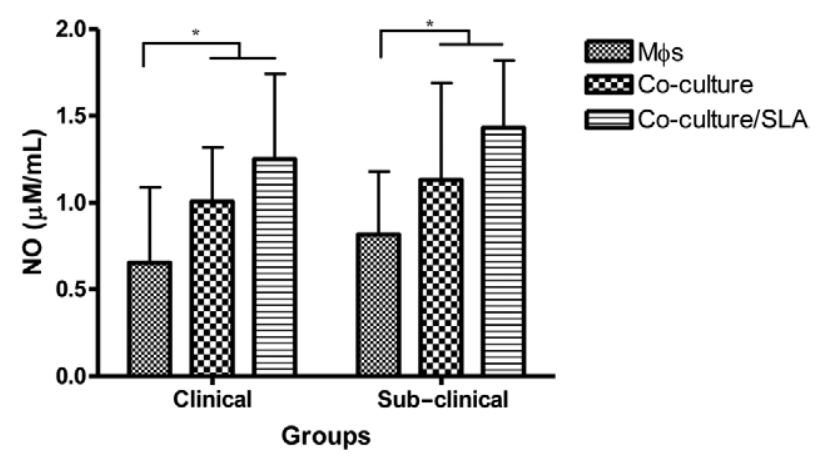

Fig. 2: nitric oxide (NO) productions by clinical and sub-clinical infected Peromyscus yucatanicus at 12 week post-infection. Two samples were analysed for each animal. Fourteen animals per group were used. Results are expressed as means \pm standard deviation. Asterisks mean $\mathrm{p}<0.05$. SLA: Leishmania antigen. 
presence or absence of SLA, but the mice were unable to clear the infection. This study is the first to document NO production by P. yucatanicus mice infected experimentally with a low or high dose of $L$. (L.) mexicana promastigotes.

As expected, none of the $P$. yucatanicus mice inoculated with $1 \times 10^{2}$ (low dose) $L$. (L.) mexicana promastigotes exhibited symptoms of the disease by the end of the study. However, the PCR was positive for $54.4 \%$ of $P$. yucatanicus mice inoculated with the low dose of $L$. (L.) mexicana promastigotes. The criteria for subclinical infection in humans include living in an area endemic for cutaneous leishmaniasis, the absence of CL symptoms or a scar and a positive Montenegro skin test (González \& Biagi 1968). The detection of Leishmania genus and subgenus kDNA by PCR must be added as a criterion for wild rodent or mouse models of subclinical leishmaniasis infection. Because P. yucatanicus is a primary reservoir of $L$. (L.) mexicana, the identification of parasite kDNA in subclinically infected animals was expected.

In contrast, $92.3 \%$ of $P$. yucatanicus mice inoculated with $2.5 \times 10^{6}$ promastigotes developed clinical infections, with lesions similar to those found in human CL patients infected with the same parasite species (Andrade-Narváez et al. 2001). This high disease rate was most likely due to the low temperature $\left(22^{\circ} \mathrm{C}\right)$. Parasite multiplication in $P$. yucatanicus was correlated with lower ambient temperature in the animal care facility in a two-year study of the persistence of $L$. (L.) mexicana in naturally infected rodents (Van Wynsberghe et al. 2000).

In laboratory studies, the control of $L$. (L.) major infection has been demonstrated to be mediated by high NO levels in a resistant M. musculus strain (C57BL/6). Moreover, the enhancer effect of autologous lymphocytes on macrophage NO production has already been demonstrated in dogs (Canis familiaris). The exposure of Leishmania (Leishmania) infantum-infected macrophages to autologous lymphocytes from dogs immunised with purified secreted antigens of $L$. (L.) infantum promastigotes resulted in a significant leishmanicidal effect due to the activation of macrophages by interferon (IFN)- $\gamma$ (Lemesre et al. 2005). Furthermore, an enhanced NOmediated antileishmanial activity of canine macrophages in response to higher IFN- $\gamma$ production by T-cells was demonstrated in co-cultures (Holzmuller et al. 2005).

In contrast, in susceptible animals (BALB/c), the exacerbation of infection is associated with the production of NO inhibitory cytokines (Trinchieri 1995, Locksley et al. 1999, Aguilar-Torrentera \& Carlier 2001, Rogers et al. 2002, Sacks \& Noben-Trauth 2002, Lang et al. 2003). CL studies that consider only the traditional model in which M. musculus mice are infected with L. major cannot address the interesting immunobiological characteristics associated with the variation among Leishmania spp. In fact, Leishmania (Leishmania) amazonensis and L. (L.) mexicana are associated with disease patterns that differ greatly from that of $L$. (L.) major in the mouse model. For example, the lesions of $\mathrm{C} 57 \mathrm{BL} / 6$ or $\mathrm{C} 3 \mathrm{H}$ mice infected with $L$. (L.) major heal, whereas these same mouse strains develop chronic disease when infected with ei- ther L. (L.) amazonensis or L. (L.) mexicana (AguilarTorrentera 2002, Pinheiro \& Rossi-Bergmann 2007). Nevertheless, NO has been considered a potent cytotoxin involved in the killing of Leishmania parasites and is therefore a key molecule in CL control (Fang 1997, Stenger et al. 1994, Bogdan et al. 2000).

Therefore, we focused on NO production by the endemic CL reservoir $P$. yucatanicus infected with $L$. (L.) mexicana from Yucatan Peninsula. In our in vitro experiments, autologous lymphocytes enhanced the NO production of macrophages collected from P. yucatanicus mice infected with either $1 \times 10^{2}$ or $2.5 \times 10^{6}$ promastigotes. This finding may be due to the release of specific cytokines, such as IFN- $\gamma$ and tumour necrosis factor alpha from lymphocytes. These cytokines exert an activating effect on Leishmania-infected macrophages and regulate NO production, as already demonstrated in the traditional mouse model.

Laboratory studies using natural hosts as experimental models could provide a better understanding of the dynamics of infection, especially concerning the ability of the immune system to address the infection. However, experiments using wild rodents are limited, primarily due to the difficulty of managing wild mammals in captivity and the absence of reagents of defined specificity available to carry out these studies.

The experimental infection of Thrichomys laurentius with Leishmania species from different complexes, $L$. mexicana and Leishmania donovani, demonstrated the ability of both Leishmania species to invade and maintain themselves in the viscera and skin of T. laurentius and no rodent displayed any lesion, histological changes or clinical evidence of infection. Nevertheless, the immune response and the dynamics of infection in this wild Leishmania reservoir are unknown (Roque et al. 2010).

The lack of a difference in the amount of NO produced by $P$. yucatanicus mice with clinical or subclinical infections indicates that as a primary reservoir of $L$. (L.) mexicana, this deer mouse maintains circulating parasites, even when subclinically infected. Leishmania is an obligate intracellular parasite of macrophages and even in humans, parasite persistence is well known (Fagundes et al. 2007, Colomba et al. 2009). Further investigation is needed to elucidate the role of the lymphoproliferative response and cytokines in the establishment of subclinical and clinical $L$. (L.) mexicana infections in P. yucatanicus mice.

Finally, the results obtained support the use of $P . y u$ catanicus as a novel experimental animal model to study CL caused by $L$. (L.) mexicana in subclinically and clinically infected mice. However, to reach this goal, it is necessary to study the expression kinetics of immune genes in $P$. yucatanicus mice infected with $L$. (L.) mexicana.

\section{ACKNOWLEDGEMENTS}

To the personnel from the animal housing facilities of the Centro de Investigaciones Regionales Dr Hideyo Noguchi, Universidad Autonoma de Yucatan, for their continuing help, to Dr Tony Schountz, for editing the paper, and to IQ Silvia Andrade Canto, for photography technical assistance. 


\section{REFERENCES}

Aguilar-Torrentera F, Carlier Y 2001. Immunological factors governing resistance and susceptibility of mice to Leishmania major infection. Rev Latinoam Microbiol 43: 135-142.

Aguilar-Torrentera F, Laman JD, Van Meurs M, Adorini L, Muraille E, Carlieri Y 2002. Endogenous interleukin-12 Is critical for controlling the late but not the early stage of Leishmania mexicana infection in C57BL/6 mice. Infect Immun 70: 5075-5080.

Alvar J, Cañavate C, Molina R, Moreno J, Nieto J 2004. Canine leishmaniasis. Adv Parasitol 57: 1-64.

Andrade-Narváez FJ, Lara SBC, Van Wynsberghe NR, RebollarTellez EA, Vargas-González A, Albertos-Alpuche NE 2003. Seasonal transmission of Leishmania (Leishmania) mexicana in the state of Campeche, Yucatan Peninsula, Mexico. Mem Inst Oswaldo Cruz 98: 995-998.

Andrade-Narváez FJ, Simmonds-Diaz EB, Aguilar-Rico S, AndradeNarváez M, Palomo-Cetina A, Canto-Lara SB, Garcia-Miss MR, Madera-Sevilla M, Albertos-Alpuche NE 1990. Incidence of localized cutaneous leishmaniasis (Chiclero's ulcer) in Mexico. Trans R Soc Trop Med Hyg 84: 219-220.

Andrade-Narváez FJ, Vargas-González A, Canto-Lara SB, DamiánCenteno AG 2001. Clinical picture of cutaneous leishmaniases due to Leishmania (Leishmania) mexicana in the Yucatan Peninsula, Mexico. Mem Inst Oswaldo Cruz 96: 163-167.

Arjona-Villicaña RD 2002. Prevalencia de infección subclínica por Leishmania en una población de alto riesgo de leishmaniosis cutánea en el estado de Campeche, MD Thesis, Universidad Autónoma de Yucatán, México, 40 pp.

Baneth G, Koutinas AF, Solano-Gallego L, Bourdeau P, Ferrer L 2008. Canine leishmaniasis-new concepts and insights on an expanding zoonosis: part one. Trends Parasitol 24: 324-330.

Bogdan C, Röllinghoff M, Diefenbach A 2000. The role of nitric oxide in innate immunity. Immunol Rev 173: 17-26.

Bretscher PA, Wei G, Menon JN, Bielefeldt-Ohman H 1992. Establishment of stable cell mediated immunity makes "susceptible" mice resistant to Leishmania major. Science 13: 342.

Canto-Lara SB, Cardenas-Marrufo MF, Vargas-González A, Andrade-Narváez F 1998. Isoenzyme characterization of Leishmania isolated from human cases with localized cutaneous leishmaniasis from the state of Campeche, Yucatan Peninsula, Mexico. Am J Trop Med Hyg 58: 444-447.

Canto-Lara SB, Van Wynsberghe NR, Vargas-González A, OjedaFarfán FF, Andrade-Narváez FJ 1999. Use of monoclonal antibodies for the identification of Leishmania spp isolated from humans and wild rodents in the state of Campeche, Mexico. Mem Inst Oswaldo Cruz 94: 305-309.

Chable-Santos JB, Van Wynsberghe NR, Canto-Lara SB, AndradeNarváez FJ 1995. Isolation of Leishmania (L.) mexicana from wild rodents and their possible role in the transmission of localized cutaneous leishmaniasis in the state of Campeche, Mexico. Am J Trop Med Hyg 53: 141-145.

Colomba C, Saporito L, Vitale F, Reale S, Vitale G, Casuccio A, Tolomeo M, Maranto D, Rubino R, Di Carlo P, Titone L 2009. Cryptic Leishmania infantum infection in Italian HIV infected patients. BMC Infect Dis 9: 199.

Doherty TM, Coffman RL 1996. Leishmania major: effect of infectious dose on T-cell subset development in BALB/c mice. Exp Parasitol 84: 124-135.

Dumonteil E, Ramirez-Sierra MJ, Escobedo-Ortegon J, Garcia-Miss MR 2003. DNA vaccines induce partial protection against Leishmania mexicana. Vaccine 21: 2161-2168.
Fagundes A, Marzochi MCA, Fernandes O, Perez MA, Schubach AO, Schubach TMP, Amendoeira MRR, Mouta-Confort E, Marzochi KBF 2007. First encounter of subclinical human Leishmania (Viannia) infection in the state of Rio Grande do Sul, Brazil. Mem Inst Oswaldo Cruz 102: 1003-1005.

Fang FC 1997. Mechanisms of nitric oxide-related antimicrobial activity. J Clin Invest 99: 2818-2825.

González HA, Biagi FF 1968. Asymptomatic infections in Mexican cutaneous leishmaniasis. Int J Dermatol 7: 8-9.

Guénet JL, Bonhomme F 2003. Wild mice: an ever-increasing contribution to a popular mammalian model. Trends Genet 19: 24-31.

Holzmuller P, Cavaleyra M, Moreaux J, Kovacic R, Vincendeau P, Papierok G, Lemesre JL 2005. Lymphocytes of dogs immunized with purified excreted-secreted antigens of Leishmania infantum co-incubated with Leishmania infected macrophages produce IFN-gamma resulting in nitric oxide-mediated amastigote apoptosis. Vet Immunol Immunopathol 106: 247-257.

Kerr S, Louise HE, Melby PC, Chang L, Perez LE, Villegas M, Miranda R 2006. Leishmania amazonensis infections in Oryzomys acritus and Oryzomys nitidus from Bolivia. Am J Trop Med Hyg 75: 1069-1073.

Lang T, Courret N, Colle J, Milon G, Antoine J 2003. The levels and patterns of cytokines produced by $\mathrm{CD} 4 \mathrm{~T}$ lymphocytes of BALB/c mice infected with Leishmania major by inoculation into the ear dermis depend on the infectiousness and size of the inoculums. Infect Immun 71: 2674-2683.

Lemesre JL, Holzmuller P, Cavaleyra M, Goncalves RB, Hottin G, Papierok G 2005. Protection against experimental visceral leishmaniasis infection in dogs immunized with purified excreted secreted antigens of Leishmania infantum promastigotes. Vaccine 23: $2825-2840$.

Locksley R, Pingel S, Lacy D, Wakil A, Bix M, Fowell D 1999. Susceptibility to infectious diseases: Leishmania as a paradigm. J Infect Dis 179: 305-308.

Menon JN, Bretscher PA 1998. Parasite dose determines the $\mathrm{Th}_{1} / \mathrm{Th}_{2}$ nature of the response Leishmania major independently of infection route and strain of host or parasite. Eur J Immunol 28: 4020-4028.

NRC - National Research Council 1996. Guide for the Care and Use of Laboratory Animals. Institute of Laboratory Animal Resources. Available from: nap.edu/openbook.php?record_id=5140.

Pinheiro RO, Rossi-Bergmann B 2007. Interferon-gamma is required for the late but not early control of Leishmania amazonensis infection in C57BL/6 mice. Mem Inst Oswaldo Cruz 102: 79-82.

Preston PM, Dumonde DC 1976. Experimental cutaneous leishmaniasis. V. Protective immunity in subclinical and self-healing infection in the mouse. Clin Exp Immunol 23: 126-138.

Rogers K, De Krey G, Mbow M, Gillespie R, Brodskyn C, Titus R 2002. Type 1 and type 2 responses to Leishmania major. FEMS Microbiol Lett 209: 1-7.

Roque AL, Cupolillo E, Marchevsky RS, Jansen AM 2010. Thrichomys laurentius (Rodentia: Echimyidae) as a putative reservoir of Leishmania infantum and L. braziliensis: patterns of experimental infection. PLoS Negl Trop Dis 4: e589.

Sacks D, Noben-Trauth N 2002. The immunology of susceptibility and resistance to Leishmania major in mice. Nat Rev Immunol 2: $845-858$

Seidelin H 1912. Leishmaniasis and babesiasis in Yucatan. Ann Trop Med Parasitol 6: 295-299.

Sosa-Bibiano EI, Van Wynsberghe NR, Canto-Lara SB, AndradeNarváez FJ 2012. Preliminary study towards a novel experi- 
mental model to study localized cutaneous leishmaniasis caused by Leishmania (Leishmania) mexicana. Rev Inst Med Trop Sao Paulo 54: 1-6.

Stenger S, Thüring H, Röllinghoff M, Bogdan C 1994. Tissue expression of inducible nitric oxide synthasis is closely associated with resistance to Leishmania major. J Exp Med 180: 783-793.

Trinchieri G 1995. Interleukin-12: a proinflammatory cytokine with immunoregulatory functions that bridge innate resistance and antigen-specific adaptive immunity. Annu Rev Immunol 13: 251-276.
Van Wynsberghe NR, Canto-Lara SB, Damián-Centeno AG, Itzá-Ortiz MF, Andrade-Narváez FJ 2000. Retention of Leishmania (Leishmania) mexicana in naturally infected rodents from the state of Campeche, Mexico. Mem Inst Oswaldo Cruz 95: 595-600.

Van Wynsberghe NR, Canto-Lara SB, Sosa-Bibiano EI, RiveroCárdenas NA, Andrade-Narváez FJ 2009. Comparison of small mammal prevalence of Leishmania (Leishmania) mexicana in five foci of cutaneous leishmaniasis in the state of Campeche, Mexico. Rev Inst Med Trop Sao Paulo 51: 87-94. 